\title{
A new perspective of refractive error calculation with mobile application
}

\author{
Komang Candra Brata, Mukhammad Sharif Hidayatulloh, Lutfi Fanani, Adam Hendra Brata \\ Multimedia, Game and Mobile Technology Research Group, Department of Computer Science, \\ Brawijaya University, Indonesia
}

\begin{tabular}{l} 
Article Info \\
\hline Article history: \\
Received Nov 20, 2019 \\
Revised Mar 9, 2020 \\
Accepted Mar 25, 2020 \\
\hline
\end{tabular}

Keywords:

Diopter

Eye health

Mobile application

Myopia

Refractive measurement

\begin{abstract}
In many situations, not standardized and limited access to eye health care in several regions of Indonesia becomes the main challenge for myopia patients to measure and monitor their current refractive error condition. Many apps were proposed to provide low-cost alternative measurement tools rather than expensive tools such as Phoropter with Snellen chart and Retinoscopy, but still, those apps need an Internet connection and manually complex steps to operate. These conditions make myopia patients reluctant to use this kind of service. In this regard, we propose an intuitive diopter level measurement app based on mobile application setup, which implements the concept of measure the user face to smartphone screen distance for the rapid diopter calculation processes and at the same time provides a low-cost alternative refractive measurement tool. This paper highlights our experiences when developing a mobile application that can help patients with myopia measuring their blur line distances and evaluate their diopter levels independently. We conduct a number of human trials with the device on a controlled environment to demonstrate the ability of the proposed app to measure the diopter level. The experimental results show that the proposed app is quite successful in measuring the diopter level of myopia patients with a relatively small range of calculation errors compared to optometrist measurement results.
\end{abstract}

Copyright () 2020 Institute of Advanced Engineering and Science. All rights reserved.

\section{Corresponding Author:}

Komang Candra Brata,

Department of Computer Science,

Universitas Brawijaya,

Veteran St., No. 8, Malang 65145, East Java, Indonesia.

Email: k.candra.brata@ub.ac.id

\section{INTRODUCTION}

Visual impairment refers to a treatable/manageable state marked by reduced vision or size of the visual field by one person. Refractive disorder is one of many forms of visual impairment which is a leading cause of visual disability throughout the world. Refractive errors are the inabilities of the eyes to focus on the visual images from the outside environment, which results in serious visual impairment in severe cases, blurred vision or even blindness [1]. The Myopia (Nearsightedness), a common form of refractive error causes vision impairment not only by direct retinal damage [2] but also by increasing the risks for cataract [3] and the onset of glaucoma [4]. For myopia patients, close objects look clear but distant objects appear blurred because the light is being focused in front of instead of on the retina of the eye [5].

The global prevalence of myopia is expected to increase from $27 \%$ of the world's population in 2010 to $52 \%$ by 2050 [6]. In the context of Indonesia as the largest archipelagic country in the world, difficulty to access eye health service is also encountered in several island regions and villages in Indonesia. In Indonesia, the regions are classified into three types, based on the difficulty of access to eye health service, namely non-remote, remote and island clusters. Non-remote regions are those which have relatively easily accessible health service and have sufficient human resources and infrastructure. Remote regions are usually 
those which have health services that are more difficult to access and have limited human resources and infrastructure. Island cluster regions are remote regions that have the most difficulties to access health service and have very limited or even non-existent human resources and infrastructure. The latest data on the prevalence of visual impairment in Indonesia is obtained through a survey on "Roadmap of Visual Impairment Control Program in Indonesia 2017-2030" which shows that the prevalence of visual impairment in Indonesia continues to be high. To overcome visual impairment in Indonesia, various efforts are needed including promotive, preventative, curative and rehabilitative action. One of the key activities in the program to manage visual impairment is to ensure the corrected vision for school-aged children and the young generation having refraction disorder and increase the availability of eye health services [7].

The diopter (D) is the unit of measurement for the refractive power of a lens. Uncorrected myopia as low as $-1.50 \mathrm{D}$ will result in moderate vision impairment, and uncorrected myopia more than $-4.00 \mathrm{D}$ is sufficient refractive error to be classified as blindness [8]. The prevailing treatment of refractive errors which are commonly used in daily life are glasses and contact lenses [9]. The common method to measure refractive error Diopter is a combination of a phoropter with a Snellen chart [10]. Phoropter contains different lenses that can be adjusted by the optometrist to get patient's edge of blur [11]. This method can gives accurate outcomes, but it still needs a large platform and can only be operated by a professional optometrist. This process also requires visual acuity knowledge from the patient as well. Retinoscopy is an alternative method of measuring refractive error which does not require patients' co-operation. This method uses a tool called a retinoscope, but this expensive tool needs the expertise to operate [12].

The emergence of smartphones makes these ubiquitous devices are increasingly complex, computationally powerful, and integrated with various sensors. A smartphone can collect and compute various data, providing opportunities to improve quality of life in a way that not easily achievable with conventional approaches [13-15]. Recent example of application which can compute the diopter digitally are evidence that this technology is feasible as portable diagnostic tools that offer an opportunity to decrease costs and increase the availability of eye healthcare for anybody, but still, those apps just digitalize a regular full examination processes or an advice of the ophthalmologist and need manual blur line distance input from the user to operate. In addition, from the user's comments and reviews, the lack of user experience in the aforementioned applications makes myopia patients reluctant to use this kind of service [16-19].

The aforementioned problems put forward for consideration that mobile applications might bring a big help for myopia patients to measure blur line distance and recognize myopia before visiting an ophthalmologist or other health services. The awareness of monitoring the eyes' health conditions is the key to prevent the myopia case from getting worse. In this paper, we explore individual responses about their awareness of eyesight problems. Next, based on collected respondent data, we develop a mobile application prototype that could compute the refractive error level automatically to investigate the feasibility of this approach. This application obviously offers an opportunity to provide a simple, low-cost and better user experience alternative measurement tool that will be a big help for myopia patients to measuring their refractive disorder level (for near-sightedness) and compute the eyeglasses power (diopter). Indirectly, it will increase the availability of eye caregivers to any myopia patients due to they can independently monitoring their eye condition by only using a smartphone.

\section{RESEARCH METHOD}

In this section, we present the development process of our minimum viable product. Considering contextual problems, we have focused on detecting the eye power of myopes. Developing automatic and intuitive diopter calculator apps for myopia has expanded our point of view about vision examination and monitoring systems, but it must be emphasized that this new approach also introduces a change in the conventional eye measurement methods which are using expensive tools to operate. Building an eye measurement application is not simply a matter of technical wizardry. This is due to the fact that calculate the power of a lens only with a smartphone needs a fancy algorithm. Additionally, develop an application that easy to use by the user is also matters. The steps for requirement elicitation for the proposed intuitive diopter calculator, computational design algorithm and system design strategies are discussed below.

\subsection{Recording user requirements}

In this paper, a diary study was used to explore and reflect on the respondent's responses to their awareness of eyesight problems. Then, based on insight from respondent data, we build a user journey to gather user requirements. A user journey map is a recently emerged method to gather requirements and designing the user experience in the application product. This map adds a third dimensional feature to a traditional user persona by focusing on a diachronic outline of a user and a product [20]. Recent works prove that this approach is an effective tool for rapidly gathering user stories in order to develop an intuitive application [21-23]. 
There can be a different number of participants actually engaged in the field study and testing. Faulkner stipulates that 20 respondents will reveal as many usability problems as more people will do and it is sufficient to offer the usability experimentation a reasonable value-to-cost balance [24]. Based on Faulkner's insight, 20 respondents were involved in this study. They live in the non-remote area were the health care facilities easy to access. They were at least familiar with Android mobile applications with minimum OS version 5 (Lollipop) and good in English. They were categorized by people with normal eyes, myopia but not wear glasses and myopia with glasses. Also, have basic knowledge or experience with measuring their current refractive error and know their own current diopter level so they can confirm the correctness of the proposed application result in the testing phase.

We are examining personal responses to their knowledge about visual acuity. During our survey and interview phase, we asked several key questions about how often they visit professional optometrist or eye care services and how they overcome their eye problems in daily life. Among all these participants, most of them said they forgot about their last visit to a doctor or an ophthalmologist because of their eye problem, 2 of them said they visited recently and all of them feel having eye problems but unwilling to went to the eye doctor.

From our studies, we have found some insights and barriers to eye health and vision examination of common people, these are:

- They are not aware of their eye problems.

- They are not visiting eye doctors or ophthalmologist regularly.

- Cost and other limitations (location, distance, transport, long queue in eye care, etc.).

Based on these insights from people who live in the non-remote area, in our understanding, the remote region's condition could present more difficulties. To overcome these difficulties, we proposed an intuitive and low cost alternative refractive error measuring tool that can be used independently by a user only with a smartphone.

\subsection{System design}

Since we are developing a mobile app to overcome the aforementioned problems, we proposed a lightweight architecture application design which has a noteworthy feature to display a diopter level to the user accurately and intuitively. This application needs to convert the raw data (the distance of user eyes and smartphone screen) into the diopter value. This application uses a front face camera to capture real-time imagery from the user faces then automatically determine the eye position to determine the distance of blur line. Next, with the distance data, the application computes the diopter level and shows the result to the user. Technically, we separate the module of the proposed app in three layers, hardware layer, face detection layer, and application layer. The hardware layer invokes the smartphones' hardware initiation, which has the main function to initiate the front camera and accelerometer control to be used as input parameters. The second layer is the face detection layer which recognizes user face and eye tracking from real-time imagery. This layer is made by integrating the Google mobile Vision API [25] in our offline module so this app technically doesn't need an Internet connection. This API provides an interface to detect the left eye, right eye, and nose as a point of interest within a face. Then based on the distance that we obtained from this process, the application layer will calculate the diopter using a formula in [26] and display the result to the user. The complete process flow is shown in Figure 1.

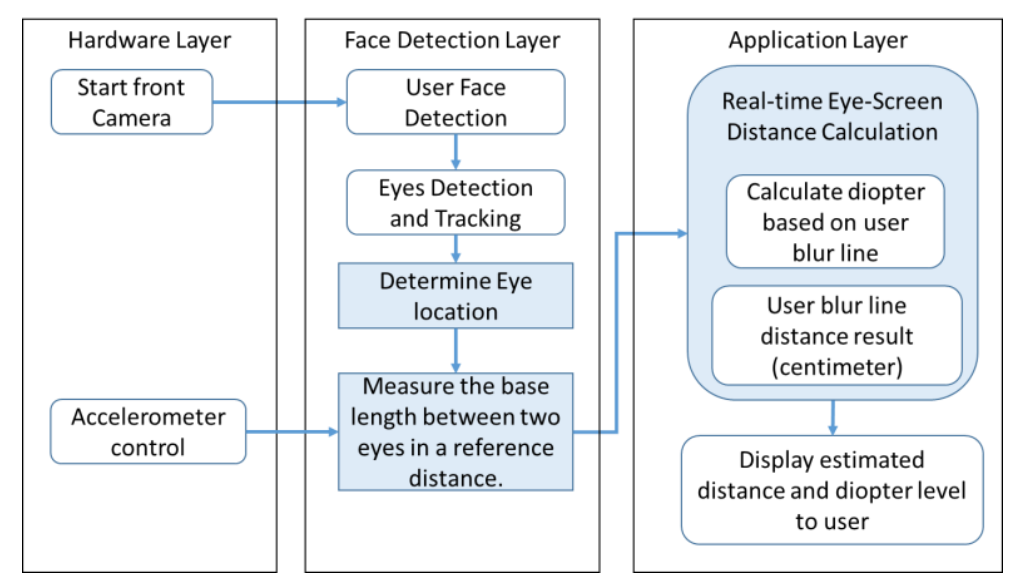

Figure 1. System module of the proposed application 


\subsection{Camera based eye to smartphone screen distance measurement}

The distance calculation of the user's face to a smartphone screen approach brings many benefits for mobile-based utility applications. Many methods have developed to calculate the distance between eye to a smartphone from using Infrared camera [27], the combination of stereo camera and infrared [28], until using video of a monocular camera [29]. Among all proposed algorithms, in 2014, Konig et al proposed an algorithm to measure the screen to face distance based on the base length data between two eyes in a reference distance (known as interpupillary distance) only using a smartphone build-in sensor and front camera. This Screen to face distance calculations output in centimeter distance [30]. Therefore, Konig's method stands out from other approaches by providing a real-time distance calculation and give natural visual experience in the way of interaction with the smartphone. The usage of the Konig's proposed application and formula to compute eyes and smartphone screen distance (f) is depicted in Figure 2.

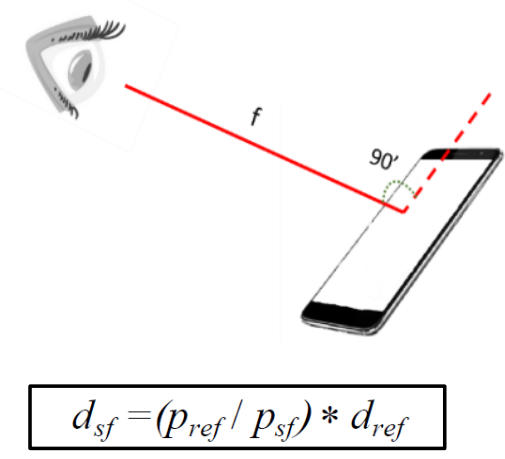

Figure 2. The design of eye-screen measurement scenario and formula to compute the distance

Here, $f$ or $(d s f)$ corresponds to the screen to face distance [cm] and dref to the interpupillary distance of the reference picture [cm], psf denotes eye to eye distance on the actual picture [pixel] and pref corresponds to the eye to eye distance on the reference picture [pixel]. This design and formula only effective when the view angle is 90 degrees from the smartphone screen.

\subsection{Compute lenses power}

We used the distance of each eye's blur line as the distance parameter. Blur line is the distance from an eye in the reading position until the text appears blurry to a person [31]. The diopter value is generated from each eye on each measurement occasion according to Algorithm 1. Konig algorithm only effective to measure the distance from 19 centimeters to 89 centimeters, this means, an application that implemented this algorithm can only effective to calculate the diopter levels from -1.12 D to -5.26D. Based on the distance that we obtain with Konig's algorithm, we calculate the diopter using the focal distance to the diopter conversion formula. Algo.1 is described in the following. First, it reads the device camera result then performs face detection which is used to process the eye tracking for a specific user eye and determine the measured eye. Next, the eye to screen distance will be computed as focal length distance and transformed to diopter level in realtime. Finally, the user only needs to move their smartphone away toward his eye to calibrate their blur line for specific eye, then the diopter will be recorded based on the user's blur line.

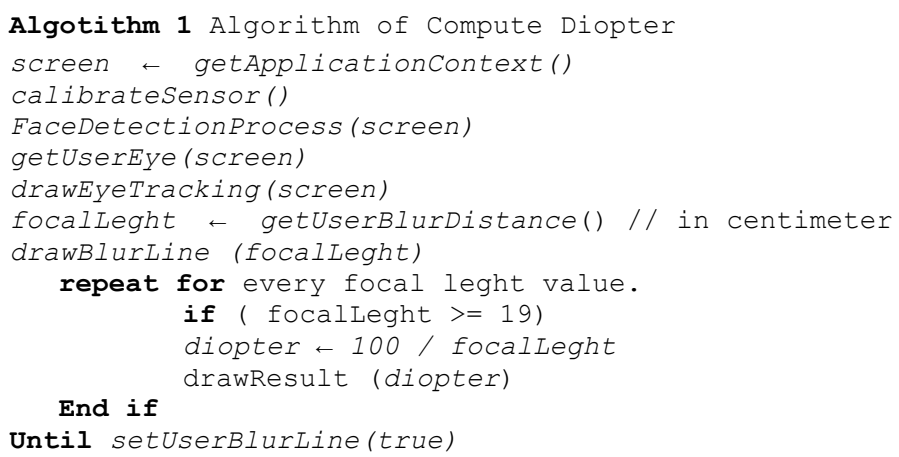




\subsection{Constructing application prototype}

The mobile application prototype was built for Android supported devices based on the insight that we have on the user journey map. The main feature of this app is to calculate the eye power to read some text in blur line area. When the user opens the application, the application provides step by step instructions to the user so they can use this application easily. Each eye may have different diopter level so this prototype is designed to calculate each eye power (right or left) start from the right eye and during the test, the other eye should remain closed. Then the application will measure the blur line distance and calculate the diopter level automatically, user only needs to adjust the smartphone until they find their blur line then tap the "record button" after they see their blur line from the screen and user will be taken to next page for the further test with consecutive eye. The screen flow for this user interaction is depicted in Figure 3.
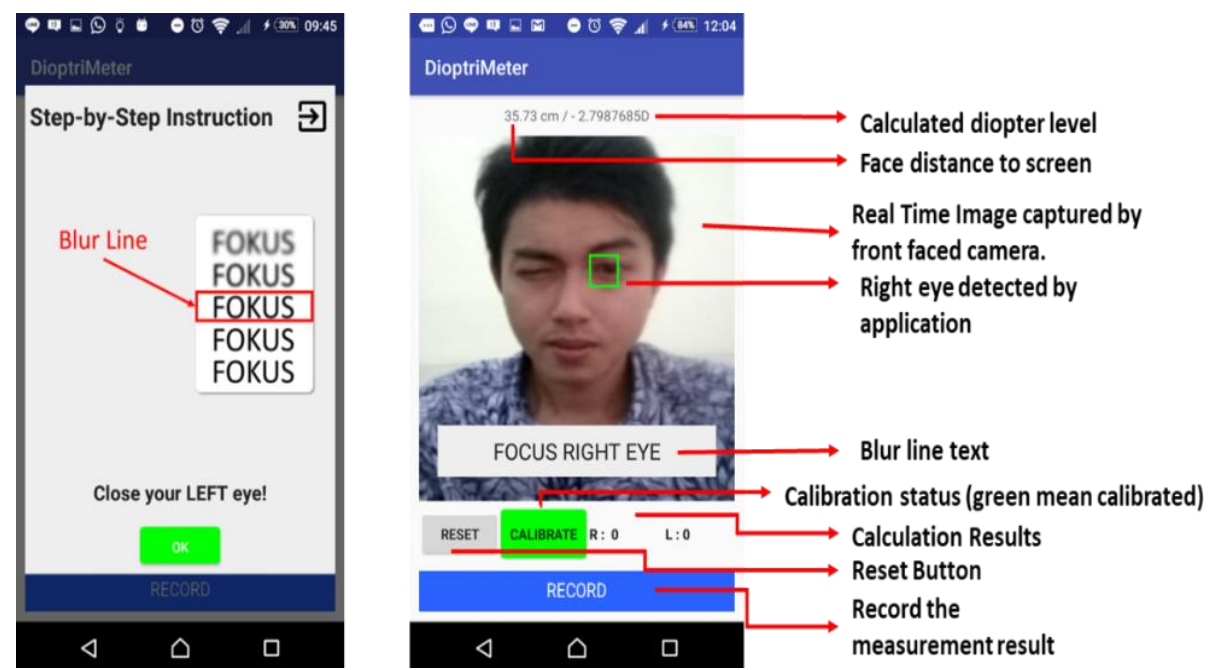

Figure 3. User interfaces of proposed application

\subsection{Prototype evaluation}

An experiment came out to understand the efficiency and ease of use of the proposed mobile application. We used usability testing to validate the usability of our approach. Usability testing is the most often utilized method for verifying and validating the quality of software [32]. The evaluation was performed with the same participants as in the user requirements elicitation phase and ask only 10 of them to do some task scenarios as described in Table 1 with our prototype. While they were performing the tasks, their performance on the tasks was observed and any problems occurred or faced by users were noted.

Table 1. Task: measure lens power

\begin{tabular}{cl}
\hline Task & Description \\
\hline 1 & Start the App \\
2 & If user wear glasses, Take off the glasses. \\
3 & Close left eye to measure the right eye blur line. \\
4 & Use the apps to automatically measure the distance from the user's eyes to the screen until getting the user's blur line. \\
5 & Use submit button to record the distance of blur line and diopter level. \\
6 & Do step 3- 5 for left eye. \\
7 & Go to the result page to see the diopter level of both eyes. \\
\hline
\end{tabular}

Usability tests were conducted using the System Usability Scale (SUS) questionnaire for the assessment of the application performance. Participants were required to rate the ten modified question items that include three usability variables: effectiveness, learnability, and satisfaction as adapted from Lewis and Sauro's research [33] with one of five responses that range from strongly agree to strongly disagree. With an overall score of 80.50 and a deviation of 4.11 , the result is quite promising, which indicates that the proposed app can easily be accepted by users [34]. Besides, participants gave positive feedback for the value and comfort provided by the instant results of the eye power tests. Next, we compare the app's results with real doctor measurement results to investigate the accuracy of the proposed application. 


\section{RESULTS AND ANALYSIS}

A direct comparison was conducted to explicitly compare the two diopter measurement process results, one with the proposed application and another measured by the doctor with a phoropter and Snellen chart. The diopter level range for each participant was recorded and the comparison result as shown in Table 2.

Table 2. The comparison result of lens power

\begin{tabular}{cccccc}
\hline No & Participant Eyes & & App (Diopter) & Doctor (Diopter) & Error Gap \\
\hline 1 & Participant 1 & R & -2.99 & -2.71 & 0.28 \\
& & L & -2.63 & -2.89 & 0.26 \\
2 & Participant 2 & R & -2.49 & -2.24 & 0.25 \\
& & L & -2.47 & -2.27 & 0.2 \\
3 & Participant 3 & R & -1.37 & -0.2 & 1.17 \\
& & L & -1.1 & -0.1 & 1.2 \\
4 & Participant 4 & R & -1.59 & -1.29 & 0.30 \\
& & L & -1.59 & -1.16 & 0.43 \\
5 & Participant 5 & R & -2.88 & -2.65 & 0.23 \\
& & L & -2.9 & -2.56 & 0.34 \\
6 & Participant 6 & R & -2.02 & -1.72 & 0.3 \\
& & L & -2.03 & -1.73 & 0.3 \\
7 & Participant 7 & R & -2.44 & -2.07 & 0.37 \\
& & L & -2.41 & -2.05 & 0.36 \\
8 & Participant 8 & R & -1.72 & -1.49 & 0.23 \\
& & L & -1.73 & -1.47 & 0.26 \\
9 & Participant 9 & R & -2.75 & -2.59 & 0.16 \\
& & L & -2.71 & -2.91 & 0.20 \\
10 & Participant 10 & R & -2.56 & -2.71 & 0.15 \\
& & L & -2.48 & -2.62 & 0.14 \\
& & Mean of Error / Deviation & & $0.2565 / 0.08$ \\
\hline
\end{tabular}

Data variability has become a prevalent issue in structured testing. The small value of standard deviation (SD) in the error gap between app measurement and doctor measurement gives information that the proposed application can give participants consistent results. As already mentioned, the evaluation result can determine whether the proposed app is satisfactory for the refractive error measurement tasks. Overall, the accuracy level gap of the proposed application is quite small which has a bit less accurate with an error of less than 0.5 Diopter (Mean=0.256, SD =0.08). Even though the proposed app has a slightly good result, it is noteworthy that there were significant deviations because the proposed app can't measure the normal eye accurately (with the error of greater than 1 Diopter). The most interesting result of our evaluation is the participant overall states that the intuitive presentation of the proposed app makes them feel easy to monitor their eye condition individually.

\section{CONCLUSION}

This paper proposes a mobile application that can measure the diopter level of a person only using front face camera and built-in smartphone sensors. The offline process of the proposed app makes it accessible for everyone even for remote area users. A user can measure the blur line distance and calculate the diopter level automatically. The general objective of the proposed application is to support the availability of eye health service for all the people in Indonesia who are in need, without being constrained by their economy and region so that each individual have opportunity to maintain their eye condition independently. The evaluation results show that the proposed prototype is quite successful in measuring the diopter level with 0.25 diopter of an error on average. This application is not intended to replace the optician's regular full examination results. The full eye test after using the application is still necessary for chronic cases. In the future, with further analysis and accuracy improvement, the proposed application could provide a big help in monitoring eye conditions to detect eye diseases and visual acuity level in their early stages.

\section{REFERENCES}

[1] M. He, et al., "Refractive error and visual impairment in urban children in southern China," Investigative ophthalmology \& visual science, vol. 45, no. 3, pp. 793-799, 2004.

[2] Group TEDC-CS, "Risk factors for idiopathic rhegmatogenous retinal detachment. The Eye Disease Casecontrol Study Group," American Journal of Epidemiology, vol. 137, no. 7, pp. 749-757, 1993. 
[3] C. Younan, et al., "Myopia and incident cataract and cataract surgery: The Blue Mountains eye study," Investigative Ophthalmology and Visual Science, vol. 43, no. 12, pp. 3625-3632, 2002.

[4] M. Qiu, et al., "Association between myopia and glaucoma in the United States population," Investigative Ophthalmology and Visual Science, vol. 54, no. 1, pp. 830-835, 2013.

[5] WH Bates, M. D., and W. Bates. The cure of imperfect sight by treatment without glasses. Mary I. Oliver, Clark Night, 2011.

[6] B. A. Holden, et al., "Global prevalence of myopia and high myopia and temporal trends from 2000 through 2050," Ophthalmology, vol. 123, no. 5, pp. 1036-1042, 2016.

[7] S. Purwati, et al., "Roadmap of Visual Impairment Control Program in Indonesia 2017-2030," The International Agency for the Prevention of Blindness (IAPB), 2018.

[8] A. G. Bennett and R. B. Rabbetts, "Bennett and Rabbetts' clinical visual optics," Butterworth-Heinemann, 1998.

[9] Rasmussen, Michael. "Overcoming risk and compliance myopia." Forrseter Research, 2006.

[10] I. A. Falkenstein, et al., "Comparison of visual acuity in macular degeneration patients measured with snellen and early treatment diabetic retinopathy study charts," Ophthalmology, vol. 115, no. 2, pp. 319-323, 2008.

[11] L. Twisselmann, "Phoropter," U.S. Patent 5223864A, 1993.

[12] F. W. Jobe, "Retinoscope," U.S. Patent 2715352A, 1955.

[13] K. C. Brata, et al., "Location-based augmented reality information for bus route planning system," International Journal of Electrical and Computer Engineering (IJECE), vol. 5, no. 1, pp. 142-149, 2015.

[14] K. C. Brata, et al., "Hanasu: Interactive Japanese Language M-Learning Application to Support Listening and Speaking Exercise," Proceedings of the 2019 3rd International Conference on Education and Multimedia Technology, ACM, pp. 311-315, 2019.

[15] L. Dennison, et al., "Opportunities and challenges for smartphone applications in supporting health behavior change: qualitative study," Journal of medical Internet research, vol. 15, no. 4, pp. 86, 2013.

[16] Innovv Script, "VEU - Myopia calculator and more," 2020. [Online] Available: https://play.google.com/store/apps/details?id=com.dayal.eyecare.free\&hl=en.

[17] Designveloper, "Eye test," $2019 . \quad$ [Online] Available: https://play.google.com/store/apps/details?id=com.b2creativedesigns.eyetest\&hl=in.

[18] Global Myopia Center, "Myopia Calculator," 2019. Available: https://globalmyopiacentre.org/myopiaresources/myopia-calculator/.

[19] J. Steiner, "End Myopia," 2019. Available: https://endmyopia.org/focal-calculator/calc.html.

[20] R. Baker, "Personas and Journey Maps," Agile UX Storytelling, Apress, Berkeley, CA, pp. 75-80, 2017.

[21] K. C. Brata, et al., "Design of public transportation navigation system on android wear device," 2017 International Conference on Sustainable Information Engineering and Technology (SIET), pp. 385-391, 2017.

[22] S. D. Reay, et al., "Prototyping collaborative relationships between design and healthcare experts: mapping the patient journey," Design for Health, vol. 1, no. 1, pp. 65-79, 2017.

[23] K. C. Brata and D. Liang, "An effective approach to develop location-based augmented reality information support," International Journal of Electrical \& Computer Engineering, vol. 9, no. 4, pp. 3060-3068, 2019.

[24] L. Faulkner, "Beyond the five-user assumption: Benefits of increased sample sizes in usability testing," Behavior Research Methods, Instruments, \& Computers, vol. 35, no. 3, pp. 379-383, 2003.

[25] Google, "Mobile Vision,” 2019. Available: https://developers.google.com/vision/.

[26] A. Lahiri, "Basic Optics: Principles and Concepts," Elsevier, 2016.

[27] M. W. Seo, et al., "Development of the viewing distance measuring device in smartphone use," 2017 39th Annual International Conference of the Engineering in Medicine and Biology Society (EMBC), pp. 1254-1257, 2017.

[28] C. E. Sutherland and T. J. Gale, "Eye-screen distance monitoring for computer use," in 2011 Annual International Conference of the IEEE Engineering in Medicine and Biology Society (EMBS), Boston, MA, pp. 2164-2167, 2011.

[29] M. T. A. Shoani, et al., "Determining subject distance based on face size," 2015 10th Asian Control Conference (ASCC), pp. 1-6, 2015.

[30] I. Konig, et al., "A new context: Screen to face distance," 2014 8th International Symposium on Medical Information and Communication Technology (ISMICT), pp. 1-5, 2014.

[31] R. J. Watt and M. J. Morgan, "The recognition and representation of edge blur: evidence for spatial primitives in human vision," Vision research, vol. 23, no. 12, pp. 1465-1477, 1983.

[32] P. Zaharias and A. Poylymenakou, "Developing a usability evaluation method for e-learning applications: Beyond functional usability," International Journal of Human-Computer Interaction, vol. 25, no. 1, pp. 75-98, 2009.

[33] J. J. Lewis and J. Sauro, "Revisiting the factor structure of the System Usability Scale," Journal of Usability Studies, vol. 12, no. 4, pp. 183-192, 2017.

[34] K. C. Brata and M. S. Hidayatulloh, "An idea of intuitive mobile diopter calculator for myopia patient," TELKOMNIKA Telecommunication Computing Electronics and Control, vol. 17, no. 1, pp. 307-313, 2019. 


\section{BIOGRAPHIES OF AUTHORS}
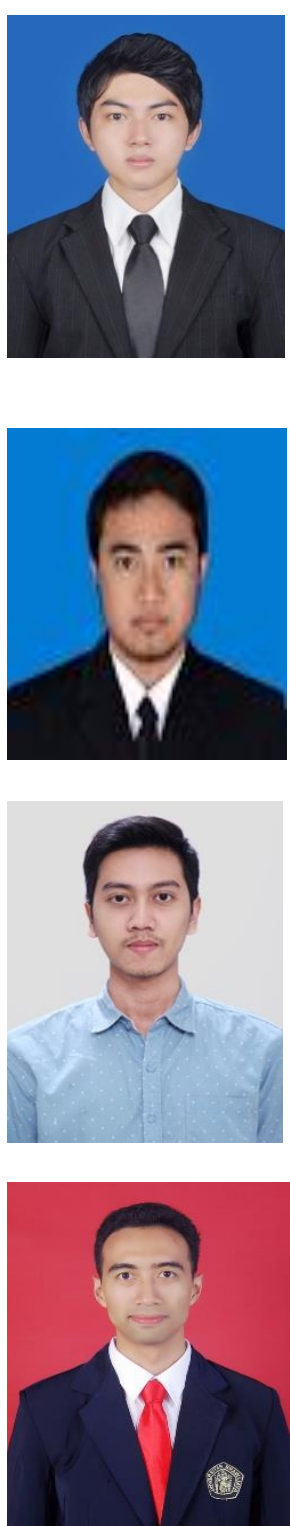

Komang Candra Brata is a lecturer in the Faculty of Computer Science, Universitas Brawijaya, Indoensia. He received the MSc degree in the Department of Computer Science and Information Engineering, National Central University, Taiwan in 2014 as an International Dual Degree Master student between the University of Brawijaya, Indonesia and National Central University, Taiwan. He completed his Bachelor's degree in the Department of Informatics Engineering, University of Brawijaya, Indonesia in 2012. His research interest area are in the areas of software engineering and information technology, user experience, HCI, mobile application development, distributed systems, and augmented reality.

Mukhammad Sharif Hidayatulloh completed his Bachelor degree in Department of Informatics Engineering, Universitas Brawijaya, Indonesia in 2017. His research interest area are in the areas of software engineering and information technology, mobile application development, and machine learning.

Lutfi Fanani is a Lecturer in the Department of Informatics Engineering, Universitas Brawijaya. He received the MSc degree in Department of Computer Science and Information Engineering, National Central University, Taiwan in 2014 as an International Dual Degree Master student between University of Brawijaya, Indonesia and National Central University, Taiwan. His research interest are in the areas of software engineering and information technology, user experience design, and mobile application development.

Adam Hendra Brata is a Lecturer in the Department of Informatics Engineering, Universitas Brawijaya. He received the MSc degree in Department of Computer Science and Information Engineering, National Central University, Taiwan in 2014 as an International Dual Degree Master student between University of Brawijaya, Indonesia and National Central University, Taiwan. His research interest is in the areas of software engineering. 